La encefalopatía espongiforme bovina y la variante de la enfermedad de Creutzfeldt-Jakob

La encefalopatía espongiforme bovina (EEB) o "enfermedad de las vacas locas" parece haber tenido su origen en el scrapie, una encefalopatía espongiforme endémica de las ovejas y cabras que se conoce en Europa desde mediados del siglo XVIII. El origen de la epidemia bovina podría explicarse por la introducción en su alimentación de harinas de origen animal producidas a partir de la trituración de las carcasas del ganado, incluido el ovino.

Aunque existen algunas dudas sobre el origen de la epidemia, que también podría deberse a la existencia de EEB endémica no reconocida, la posibilidad de que la fuente de la infección fueran las harinas de origen animal ha llevado a la adopción en el Reino Unido de una serie de medidas destinadas a romper el ciclo de la reinfección de los bovinos, a limitar la extensión geográfica de la enfermedad y a eliminar posibles fuentes de nuevas infecciones. La más importante probablemente haya sido la prohibición en 1988 de la administración de estos suplementos proteínicos a los rumiantes, que permitió empezar a controlar la epidemia hacia 1992.

La EEB no está limitada al Reino Unido. En algunos países, entre ellos este último, está disminuyendo la incidencia, pero en otros, como Alemania, España, Francia, Irlanda o Portugal, parece estar aumentando o han empezado a aparecer recientemente los primeros casos, hecho que podría explicarse por una mejor identificación de los casos, basada en la vigilancia activa y los métodos inmunológicos. Hasta ahora no se ha identificado ningún caso índice nativo fuera del Reino Unido.

Aunque en otros países se produjeron cambios en la alimentación del ganado similares a los del Reino Unido, aparentemente la EEB solo ha aparecido en este país. La explicación más verosímil es que la proporción de ovinos en las harinas animales y la proporción de ovinos infectados por el scrapie fueran mayores en el Reino Unido que en cualquier otro país. Otra posibilidad es que en los años 70 se hubiera producido una mutación patógena en los bovinos del Reino Unido. Sin embargo, en los humanos se han producido mutaciones en todo el mundo y no hay motivos para pensar que en este aspecto los humanos difieran del resto de los mamíferos.

A las pocas semanas de la identificación del primer caso de EEB empezó a manifestarse la preo- cupación de que la enfermedad pudiera afectar a los humanos y se empezaron a tomar medidas para evitar que los tejidos potencialmente infectados entraran en la cadena alimentaria humana. En mayo de 1990 se estableció en el Reino Unido una unidad de vigilancia para monitorear la ECJ y a los 3 años se extendió a otros países europeos, bajo la coordinación de la Unión Europea. A pesar de que en los 10 años posteriores a la identificación del primer caso de EEB, los casos de ECJ no aumentaron en los grupos en riesgo y siguieron presentando las mismas características clínicas y neuropatológicas que antes de la aparición de la epidemia de EEB, entre mayo y octubre de 1995 se notificaron en el Reino Unido tres casos de ECJ en pacientes jóvenes con una característica neuropatológica inesperada: la presencia de placas amiloides.

Con la aparición de cinco nuevos casos similares en ese país empezó a conformarse un nuevo síndrome clínico asociado con la formación de placas y caracterizado por inicio en edades jóvenes, síntomas psiquiátricos tempranos, marcada ataxia, ausencia de actividad electroencefalográfica periódica y duración relativamente prolongada de la enfermedad. Además, el examen neuropatológico comparativo de los casos históricos con estos casos recientes confirmó que eran realmente distintos. Todos presentaban placas morfológicamente inusuales, denominadas floridas o "en margarita", en las que el núcleo de amiloide estaba rodeado por "pétalos" espongiformes. Aunque el análisis genético de seis de estos ocho casos no permitió identificar mutaciones patógenas, su comparación con otros casos de ECJ en jóvenes de otros países europeos reveló que ninguno de estos presentaba las características clínicas y neuropatológicas de los casos británicos. Un informe de 10 casos británicos concluyó que estábamos ante una variante antes desconocida de ECJ que ocurría únicamente en menores de 45 años y que probablemente se debía a la exposición a la EEB.

Esta relación está establecida actualmente de forma convincente, después de que los estudios de laboratorio demostraran que los patógenos aislados del ganado con EEB y de los casos humanos de ECJ presentaban las mismas características biológicas y moleculares distintivas. La fuente de la contaminación parece haber sido la carne de ternera, aunque nunca se ha podido demostrar que el músculo contenga el agente infeccioso en ninguna forma de encefalopatía espongiforme en ninguna de las espe- 
cies afectadas. Por lo tanto, lo más probable es que la infección se deba a productos contaminados por tejido del sistema nervioso central.

Aunque la cantidad de tejido infeccioso ingerido debe ser un importante factor determinante de la transmisión de la EEB al ser humano en forma de ECJ, el genotipo humano en el codón polimórfico 129 del gen PRNP parece desempeñar un papel importante en la susceptibilidad a la infección. Las alternativas metionina/valina, metionina/metionina y valina/valina están distribuidas en la población caucasoide en proporciones aproximadas de 50, 40 y $10 \%$ y todos los pacientes con vECJ (76) han sido homocigóticos para la metionina. Sin embargo, también es posible que, como ocurre en el kuru y en la ECJ yatrógena, los individuos heterocigóticos sean comparativamente resistentes a la enfermedad y solo la manifiesten tras períodos de incubación superiores a los de individuos homocigóticos.

A diferencia de la epidemia de EEB, el brote de ECJ ha mostrado un pequeño incremento en los primeros 6 años, diferencia que podría deberse al hecho de que en los humanos no ha habido reciclaje de los tejidos infectados o a los pequeños inóculos ingeridos, que serían insuficientes para producir la infección, excepto en individuos genéticamente susceptibles. La incertidumbre sobre la magnitud del brote de ECJ se debe al desconocimiento del período de incubación de la enfermedad. Si fuera de 10 a 15 años podría esperarse un importante aumento del número de casos de ECJ en los próximos años, pero si fuera de 5 a 10 años, dicho número seguiría siendo escaso, gracias a las medidas adoptadas entre 1987 y 1997 para limitar la exposición de los humanos y de los animales a la EEB. Dependiendo del período de incubación y de otras variables, los modelos matemáticos predicen que la magnitud del brote podría variar desde menos de un centenar de casos hasta cientos de miles.

Si hay un gran número de personas incubando silenciosamente la enfermedad, el riesgo de transmisión yatrógena humana de la ECJ es serio y muy superior al de la ECJ esporádica.

Varios gobiernos han puesto en práctica medidas para minimizar el riesgo de transmisión entre humanos a través de las donaciones de sangre. En el Reino Unido, todo el plasma es importado y toda la sangre de donantes británicos es filtrada para eliminar los leucocitos, que son las células de la sangre con mayores probabilidades de ser portadoras de la infección. En los Estados Unidos de América se han excluido las donaciones de cualquiera que haya vivido en el Reino Unido durante un período acumulado de más de 6 meses entre 1980 y 1996, y lo mismo han hecho otros países, como Alemania, Australia, Canadá, Japón, Nueva Zelandia y Suiza.
Teniendo en cuenta la posibilidad de que la infección esté muy extendida en el Reino Unido, la preocupación está justificada en relación no solo con las donaciones de sangre y órganos, sino también con el uso de instrumental médico y quirúrgico, particularmente del utilizado en neurocirugía y cirugía ocular. Sin embargo, en ausencia de una prueba de detección, es imposible implantar una política encaminada a eliminar todo riesgo, pues eso requeriría la paralización del programa nacional de donación de órganos. Lo mismo se aplica a los procedimientos médicos cruentos y a los procedimientos quirúrgicos, que no se pueden suspender por el riesgo teórico de ECJ ni se pueden denegar a los individuos con sospecha de padecer de ECJ. En estas condiciones, se debería utilizar instrumental desechable siempre que fuera posible e implantar un protocolo estandarizado y estricto de esterilización del instrumental reutilizable. Otro problema igualmente importante radica en la posibilidad de que el agente del scrapie adaptado a los bovinos haya vuelto a cruzar la barrera de especies hacia las ovejas, llevando consigo su nueva capacidad de infectar a los humanos. (Brown P, Will RG, Bradley R, Asher DM, Detwiler L. Bovine spongiform encephalopathy and variant CreutzfeldtJakob disease: background, evolution, and current concerns. Emerg Infect Dis 2001;7:6-16.)

\section{Serotipos de Chlamydia trachomatis y riesgo de carcinoma cervical de células escamosas}

La infección por papilomavirus humanos (PVH) es la principal causa de neoplasia cervicouterina. Otros factores de riesgo son otras enfermedades de transmisión sexual y el consumo de tabaco. Los estudios seroepidemiológicos longitudinales han proporcionado pruebas de que la infección por Chlamydia trachomatis constituye un factor de riesgo adicional de carcinoma cervical invasor de células escamosas (CCCE). Después de identificar una relación entre la presencia de anticuerpos séricos contra $C$. trachomatis y el posterior desarrollo de CCCE, este grupo de investigadores escandinavos se propuso determinar si esta asociación se limitaba a ciertos serotipos.

Los casos de carcinoma cervical se identificaron cruzando los datos de tres bancos de suero de Finlandia, Noruega y Suecia con los datos de tres registros oncológicos de estos mismos países. A finales de 1994 se habían identificado 181 casos de carcinoma cervical invasor confirmado histológicamente que fueron apareados por edad ( \pm 2 años), tiempo de almacenamiento de las muestras $( \pm 2$ meses) y país de origen con tres controles sin cáncer cervical en el momento del diagnóstico de los casos. 
Las muestras de suero de 10 controles tenían un volumen insuficiente, por lo que solo se estudiaron 533 controles, en vez de los 543 previstos.

Los anticuerpos séricos de la clase IgG contra C. trachomatis y C. pneumoniae se determinaron mediante microinmunofluorescencia (MIF). Se consideraron positivos los títulos de 1:16 para C. trachomatis y de 1:32 para C. pneumoniae, que se utilizó como antígeno de control. La IgG sérica frente a $C$. trachomatis también se midió mediante dos tipos de ensayos inmunoenzimáticos (ELISA): uno para cuerpos elementales y otro para péptidos de la principal proteína de la membrana externa (MOMP). Todas las muestras de suero con anticuerpos frente a C. trachomatis fueron analizadas también mediante MIF para detectar anticuerpos específicos contra los serotipos B, C, D, E, F, G, H, I y J de C. trachomatis. En 85 casos (47\%) también se procedió a la amplificación (reacción en cadena de la polimerasa) del ADN extraído de las muestras de biopsia para detectar la presencia de ADN de C. trachomatis mediante la prueba automatizada Cobas Amplicor.

Los datos fueron analizados teniendo en cuenta los efectos de los otros dos principales factores de riesgo de CCCE: la infección por PVH y el consumo de tabaco. Para ello se determinaron los títulos de anticuerpos IgG frente a los PVH 16, 18 y 33 mediante ELISA, y las concentraciones de cotinina mediante radioinmunoensayo. Se calcularon las razones de los productos cruzados (OR) y los intervalos de confianza del 95\% (IC95\%).

De los 181 carcinomas, $109(60 \%)$ estaban localizados y $62(34 \%)$ eran mestastásicos. En los otros 10 casos (6\%) se desconocía el estadio; 150 eran CCCE. Las pacientes tenían un promedio de 44 años (23-64) en el momento del diagnóstico y el intervalo medio entre la donación del suero y el diagnóstico fue de 56 meses (1-221).

La prevalencia general de anticuerpos séricos IgG frente a $C$. trachomatis fue de $27 \%$ en los casos y $13 \%$ en los controles. Las tasas correspondientes de anticuerpos frente a los tres tipos de $\mathrm{PVH}$ fueron de 37 y $18 \%$, y para la cotinina, de 50 y $39 \%$. En general, las dos pruebas de ELISA fueron más sensibles que la MIF. Las OR de carcinoma cervical ajustadas para la presencia de PVH y cotinina fueron menores con las pruebas de ELISA (1,5; IC95\%: 1,0-2,4 con la prueba para cuerpos elementales y 1,3; IC95\%: $0,9-1,9$ con la prueba para péptidos) que con la MIF (1,8; IC95\%: 1,1-2,8). La presencia de IgG sérica frente a $C$. pneumoniae no se asoció con el carcinoma cervical (OR ajustada: 1,2; IC95\%: 0,8-1,7).

Las mayores estimaciones del riesgo correspondieron a los casos de CCCE diagnosticados $12 \mathrm{o}$ más meses después de la donación de suero. En estos 128 casos el serotipo G fue el más estrechamente asociado al CCCE (OR ajustada: 6,6; IC95\%:
1,6-27,0). Otros serotipos asociados con el CCCE fueron el I (OR: 3,8; IC95\%: 1,3-11,0) y el D (OR: 2,7; IC95\%: 1,3-5,6). El serotipo B mostró una asociación casi significativa (OR: 4,1; IC95\%: 1,0-18,0). La exposición a más de un serotipo incrementó significativamente el riesgo de CCCE. La reacción en cadena de la polimerasa detectó la presencia de $\mathrm{ADN}$ de $C$. trachomatis en $4(5 \%)$ de los 79 casos analizados. Uno era seropositivo para múltiples serotipos (D, E, F, H, I, J), y los otros tres eran seronegativos.

Según los autores, este estudio es el primero que proporciona pruebas seroepidemiológicas longitudinales de la existencia de una asociación entre la exposición a serotipos específicos de C. trachomatis y el CCCE. El mayor riesgo se asoció con la presencia de IgG sérica frente al serotipo G, y la presencia de anticuerpos frente a más de un serotipo incrementó el riesgo.

Las diferencias entre serotipos detectadas en este estudio y el hecho de que el riesgo fuera mayor en las mujeres expuestas a más de un serotipo son datos que apuntan hacia la participación de C. trachomatis en la carcinogénesis cervical. (Anttila T, Saikku P, Koskela P, Bloigu A, Dillner J, Ikäheimo I, et al. Serotypes of Chlamydia trachomatis and risk for development of cervical squamous cell carcinoma. JAMA 2001;285:47-51)

\section{Eficacia de la vacunación masiva contra la enfermedad por meningococos del grupo C en Québec}

A finales de los años 80 se registró en Québec un aumento del número de casos de enfermedad por meningococos (EMC), asociado con un aumento de la proporción de casos causados por un clon virulento de Neisseria meningitidis serogrupo C serotipo 2a, con un aumento de la proporción de casos en adolescentes y adultos jóvenes y con altas tasas de letalidad y secuelas. Para controlar este brote, en 1991 y 1992 se realizaron programas de vacunación dirigidos a los niños en edad escolar y a los adolescentes, pero la incidencia de EMC del serogrupo $C$ siguió siendo elevada en los grupos no vacunados. En consecuencia, las autoridades decidieron iniciar un programa para la vacunación masiva y gratuita de los 1,9 millones de habitantes de esta provincia canadiense entre los 6 meses y 20 años de edad. La campaña, iniciada en diciembre de 1992, había concluido en marzo de 1993 y, en total, se distribuyeron aproximadamente 1,6 millones de dosis de diferentes vacunas: tetravalente $(\mathrm{A}$, C, Y, W135) de Connaught, North New York, Ontario ( $24 \%$ de las dosis), bivalente (A, C) de SmithKline-Beecham, Rixensart, Bélgica (4\%) y bivalente (A, C) de Mérieux, Lyon, Francia (72\%). En este es- 
tudio se analiza el efecto de este programa de vacunación masiva sobre la epidemiología de la EMC y la eficacia a medio plazo de la vacuna polisacárida en función de la edad de vacunación.

La incidencia de la EMC en la población se analizó en el período comprendido entre 1990 y 1998. Para determinar la eficacia de la vacuna se comparó la incidencia de EMC del serogrupo C confirmada por cultivo entre los vacunados y los no vacunados. El período fue del 1 de abril de 1993 al 31 de marzo de 1998. En el grupo de control, la "edad de vacunación" se calculó como la diferencia entre el 30 de junio del año de nacimiento y el 1 de febrero de 1993, punto mediano de la campaña de vacunación masiva. La eficacia de la vacuna se definió como 1 menos el riesgo relativo de la enfermedad y se calcularon los intervalos de confianza del 95\% (IC95\%) de la eficacia de la vacuna, ajustada o no en función de la edad. El número total de casos prevenidos entre los vacunados se calculó como la suma de las diferencias entre los números observados y esperados en cada año de edad.

Entre el 1 de enero de 1990 y el 31 de diciembre de 1998 se registraron 899 casos de EMC: 355 del serogrupo C; 332 del serogrupo B; 36 del serogrupo, $\mathrm{Y} ; 17$ de otros serogrupos y 10 de serogrupos indefinibles. En otros 71 casos no se procedió a la identificación del serogrupo y no existía información sobre los 78 casos restantes. La incidencia de EMC del serogrupo C disminuyó marcadamente en abril de 1993, después de la campaña de vacunación masiva, y siguió siendo baja a partir de entonces. Después de ajustar las tasas en función de los casos en los que no se procedió a la identificación del serogrupo, la incidencia anual media de EMC del serogrupo C fue de 1,4 por 100000 en 1990-1992 y de 0,3 por 100000 en 1993-1998. No hubo un aumento de la incidencia de casos debidos a todos los demás serogrupos, que fue exactamente la misma $(0,7$ por 100 000) en ambos períodos. Sin embargo, hubo un pequeño aumento de la proporción de casos del serogrupo $Y$, con una media de 1 caso por año en 1990-1992 y de 5,5 casos por año en 1993-1998.

La tasa media de vacunación fue de $84 \%$, con una cobertura ligeramente menor en los niños pequeños, y marcadamente menor en los de 17 años o más. Entre el 1 de abril de 1993 y el 31 de marzo de 1998 se identificaron 53 casos de EMC del serogrupo C: 14 en los individuos no vacunados y 39 en los vacunados, de los cuales tres recibieron la vacuna a principios de 1992 y los demás durante la campaña de vacunación masiva. Entre los casos en los que la vacunación fracasó, el intervalo mínimo entre su administración y el inicio de la enfermedad fue de 46 días.

El riesgo de EMC del serogrupo $C$ fue mayor en los primeros 2 años posteriores a la vacunación masiva, período en el cual fue más elevado en los individuos no vacunados que en los vacunados. Entre el tercero y el quinto año el riesgo disminuyó en ambos grupos. En los individuos no vacunados, la tasa de incidencia de ECM del serogrupo $C$ fue de 1,78 por 100000 años-persona (IC95\%: 0,89 a 3,19) durante los primeros 2 años y de 0,32 (IC95\%: 0,07 a 0,95 ) entre el tercero y el quinto año. En los vacunados, las cifras correspondientes fueron 0,62 (IC95\%: 0,38 a 0,95) y 0,39 (IC95\%: 0,24 a 0,61), respectivamente. La eficacia no ajustada de la vacuna fue de $47 \%$ (IC95\%: $-6 \%$ a $72 \%$ ) a lo largo de los 5 años, de $65 \%$ (IC95\%: $20 \%$ a $84 \%$ ) durante los primeros 2 años, y de $0 \%$ (IC95\%: $-5 \%$ a $65 \%$ ) del tercero al quinto año.

Mediante regresión logística se observó una estrecha relación entre la eficacia de la vacuna y la edad de vacunación: 83\% (IC95\%: 39\% a 96\%) entre los 15 y los 20 años, 75\% (IC95\%: -17\% a 93\%) entre los 10 y los 14 años, y 41\% (IC95\%: $-106 \%$ a $79 \%$ ) entre los 2 y los 9 años. En los menores de 2 años no se demostró ningún efecto protector; los 8 casos registrados en este grupo de edad ocurrieron en niños vacunados. La eficacia de la vacuna solo fue significativa en el grupo de 15 a 20 años. En total, se estimó que la campaña de vacunación masiva previno 48 casos de EMC durante los 5 años (1 caso por 34000 dosis).

Los autores destacan que este estudio es el primero que analiza el impacto a medio plazo de una campaña de vacunación masiva contra la EMC del serogrupo $C$ en una población amplia y bien definida. Sus resultados les llevan a concluir que la vacunación masiva es eficaz para controlar los brotes de EMC producidos por el serogrupo $C$ en adolescentes y adultos jóvenes, y que se observa protección durante los primeros 2 años posteriores a la vacunación. El escaso número de casos registrados en los 3 años siguientes no permitió llegar a conclusiones firmes, pero es de esperar que la eficacia de la vacuna disminuya con el tiempo. (De Wals P, De Serres G, Niyonsenga T. Effectiveness of a mass immunization campaign against serogroup $\mathrm{C}$ meningococcal disease in Quebec. JAMA. 2001; 285:177-181.)

\section{Plaguicidas y salud pública: métodos integrados de control de los mosquitos}

Este artículo analiza el futuro del uso de los plaguicidas como parte de los métodos integrados de control de los mosquitos.

Las enfermedades transmitidas por vectores, entre los que se encuentran varios mosquitos, constituyen un importante problema internacional de salud pública. 
En los Estados Unidos de América (EE.UU.) el control de los mosquitos ha evolucionado de la aplicación de insecticidas a los programas integrados de control de plagas, que incluyen la vigilancia, la reducción de las fuentes, los larvicidas, el control biológico y la educación pública. No obstante, los adulticidas siguen desempeñando un importante papel en caso de inundaciones o de brotes de enfermedades como la encefalitis de San Luis. Los principios del control integrado de mosquitos están disponibles en la Internet (http://www.ifas.ufl.edu/ $\sim$ pest/vector).

Una vez que los programas de vigilancia confirman que las poblaciones de mosquitos (larvas o adultos) han excedido determinado umbral, se inician las actividades de control. La reducción de las fuentes consiste en eliminar los hábitat de las larvas o en hacerlos inadecuados para su desarrollo. La educación pública es un elemento importante de dicha reducción. El control biológico incluye el empleo de diferentes predadores (ninfas de libélula y otros invertebrados, peces insectívoros, hongos entomopatógenos, como Laginidium giganteum, o nemátodos entomoparasitarios, como Romanomermis culicivorax y R. iyengari). Las trampas para mosquitos han sido utilizadas durante años para monitorear las poblaciones de estos insectos y últimamente se han desarrollado nuevos diseños. Las trampas eléctricas de alto voltaje con luz negra o ultravioleta no proporcionan un control satisfactorio de los mosquitos y matan indiscriminadamente a otros insectos.

Los plaguicidas utilizados por los organismos estatales o locales tienen advertencias e instrucciones de uso para reducir al mínimo los riesgos para la salud humana y para el medio ambiente. Estos plaguicidas son aplicados por empleados públicos entrenados específicamente para seguir estas instrucciones. Los insecticidas contra los mosquitos son tóxicos para los pájaros, peces, invertebrados acuáticos y abejas. La exposición a los insecticidas de animales a los que no están destinados es limitada, aunque puede ocurrir. La exposición humana en áreas residenciales también es rara, siempre que se tomen las medidas adecuadas. También existe el riesgo de que el viento lleve los plaguicidas hacia los cultivos.

En áreas donde no es factible el control biológico ni la reducción de las fuentes, la detección de gran número de mosquitos inmaduros puede requerir la aplicación de larvicidas para evitar la emergencia de los mosquitos adultos. El uso de larvicidas es menos controvertido que el de los adulticidas, aunque puede tener efectos adversos sobre artrópodos y vertebrados acuáticos.

A pesar de las ventajas de los programas integrados, por falta de presupuesto y personal, algunos programas locales de control de mosquitos tienen que depender únicamente de los adulticidas. Para que sean eficaces, los insecticidas tienen que ser aplicados en gotículas que se mantengan en el aire; gotas mayores que caigan al suelo o sobre la vegetación pueden causar efectos indeseables en organismos a los que no están destinadas, además de constituir un desperdicio. Para conseguir esas gotículas se utilizan equipos especiales. La fumigación aérea suele producir gotículas de 30 a $50 \mu \mathrm{m}$ con menos de $2,5 \%$ de gotículas de diámetro superior a $100 \mu \mathrm{m}$, y la fumigación terrestre con métodos de volumen ultra-bajo (ULV), gotículas de 8 a $30 \mu \mathrm{m}$, sin que ninguna supere los $50 \mu \mathrm{m}$. El viento es necesario para dispersar las gotículas, pero si su velocidad es excesiva reduce el control. Los mosquitos adultos se controlan fácilmente con la aplicación de insecticidas a concentraciones extremadamente bajas. Por ejemplo, el malatión se aplica a razón de 219,8 mL/ha para controlar los mosquitos, mientras que en la agricultura se aplica a razones de hasta $1172 \mathrm{~mL} / \mathrm{ha}$.

La resistencia de los vectores a algunos larvicidas y adulticidas ha ocurrido periódicamente. La resistencia debe ser confirmada mediante pruebas de laboratorio para excluir otros factores como la inadecuada calibración de los equipos, otros errores de aplicación y factores climáticos.

Puede haber resistencia cruzada a insecticidas de una misma clase y diferentes especies de mosquitos pueden tener diferente susceptibilidad a distintos larvicidas y adulticidas. Para evitar el desarrollo de resistencia se pueden alternar insecticidas con diferentes modos de acción. Sin embargo, solo existen dos clases químicas de adulticidas con diferente modo de acción: los organofosforados y los piretroides. Aunque existan controles biológicos, incluidos los pájaros y los murciélagos, su número suele ser insuficiente para proporcionar un control alternativo adecuado. Por lo tanto, el control continuo de los mosquitos requiere el uso alternante de diferentes clases de insecticidas, junto con el monitoreo de la resistencia, la reducción de las fuentes, el control biológico y la educación del público.

Los repelentes, en particular la $\mathrm{N}, \mathrm{N}$-dietilmetatoluamida (DEET), se utilizan para evitar las picaduras de los mosquitos y de otros insectos y pueden ayudar a reducir la transmisión de enfermedades, aunque no son suficientes. Además, algunos pueden ser ineficaces.

En la última década ha habido un marcado aumento de la preocupación de los ciudadanos por el uso de plaguicidas, a pesar de que hay normas bien establecidas para evaluar y mitigar sus riesgos para los humanos y para el medio ambiente. El artículo concluye con un análisis de las leyes estadounidenses sobre los plaguicidas usados en salud pública. (Rose RI. Pesticides and public health: integrated methods of mosquito management. Emerg Infect Dis 2001;7:17-23.) 